\title{
Speed of visual processing increases with eccentricity
}

\author{
Marisa Carrasco $^{1,2}$, Brian McElree ${ }^{1}$, Kristina Denisova $^{1}$, and Anna Marie Giordano ${ }^{1}$ \\ ${ }^{1}$ Department of Psychology, New York University, 4 Washington Place, New York, New York \\ 10003, USA. \\ ${ }^{2}$ Department of Neural Science, New York University, 4 Washington Place, New York, New York \\ 10003, USA.
}

\section{Abstract}

The visual system has a duplex design to meet conflicting environmental demands: the fovea has the resolution required to process fine spatial information, but the periphery is more sensitive to temporal properties $1^{-} 3$. To investigate whether the periphery's sensitivity is partly due to the speed with which information is processed, we measured the full timecourse of visual information processing by deriving joint measures of discriminability and speed, and found that speed of information processing varies with eccentricity: processing was faster when same-size stimuli appeared at $9^{\circ}$ than $4^{\circ}$ eccentricity, and this difference was attenuated when the $9^{\circ}$ stimuli were magnified to equate cortical representation size. At the same eccentricity, larger stimuli are processed more slowly. These temporal differences are greater than expected from neurophysiological constraints $4^{-} 6$.

The main projection from the retina to the cerebral cortex is dominantly organized into two parallel pathways: magnocellular $(\mathrm{M})$ and parvocellular $(\mathrm{P})$. Visual information is relayed from the retina through the $\mathrm{M}$ and $\mathrm{P}$ divisions of the lateral geniculate nucleus and of striate cortex. Although the two pathways are not completely segregated in the visual cortex, their spatiotemporal characteristics are thought to have perceptual consequences4. In macaque monkeys and cats, speed of conduction and integration time are about $20 \mathrm{~ms}$ faster for $\mathrm{M}$ than for P neurons5, 6 , and the ratio of P:M cells decreases with eccentricity7. To our knowledge, no neurophysiological study has directly assessed how latency varies with eccentricity. Given the similarities of human and nonhuman primates' visual systems, these neurophysiological constraints might result in faster processing of visual stimuli in humans in peripheral than in parafoveal regions. Finding faster response time to peripheral stimuli8 would not settle the question, however. Response time differences can be due to changes in discriminability or in decision criteria across conditions, which can lead to speed-accuracy tradeoffs. To control for these tradeoffs, we used the response signal speed-accuracy tradeoff (SAT) procedure, which provides conjoint measures of discriminability and information accrual9 ${ }^{-} 11$.

In a two-alternative, forced-choice discrimination task, three naive observers performed an orientation feature search (Fig. 1a). They indicated whether a target was tilted $30^{\circ}$ to the right or to the left. The target, a 2-c.p.d. Gabor patch (suprathreshold sinusoidal grating

(C) 2003 Nature Publishing Group

Correspondence should be addressed to M.C. (marisa.carrasco@nyu.edu).

COMPETING INTERESTS STATEMENT

The authors declare that they have no competing financial interests.

Note: Supplementary information is available on the Nature Neuroscience website. 
vignetted by a Gaussian envelope subtending $2^{\circ}$ of visual angle), appeared at $4^{\circ}$ or $9^{\circ}$ eccentricity by itself or among seven vertical 2-c.p.d. Gabor patches (distracters). A cue indicated the display onset (see Supplementary Methods online). To sample the full timecourse of processing, from early times when discrimination was at or near the chance level to late times when it had reached its asymptotic level, a tone sounded at 1 of 7 times ranging from 40 to $2,000 \mathrm{~ms}$ after the display onset. To illustrate how this procedure provides measures of both discriminability and processing dynamics, we plotted discrimination performance as a function of processing time in hypothetical SAT functions (Fig. 1b).

The timecourse data (within and across subjects; $n=3$ ) were fit with an exponential approach to a limit:

$$
d^{\prime}(t)=\lambda\left(1^{-} e^{-\beta(t-\delta)}\right), \text { for } t>\delta, \text { else } 0
$$

We used a hierarchical model-testing scheme to determine how eccentricity and set size affected the three parameters of equation (1), which provides a quantitative summary of the shape of the SAT function9-11. In equation (1), discriminability with maximal processing time is estimated by the asymptotic parameter $\lambda$, and speed of processing is estimated by two parameters: $\delta$, the intercept parameter specifying the time when discriminability departs from chance $\left(d^{\prime}=0\right)$, and $\beta$, the rate parameter indexing the speed with which discriminability grows from chance to asymptote. We show the parameter estimates of the best fitting models for the average data for all conditions, based on fits of nested models to the individual observers' data that systematically varied the three parameters of equation (1) (Fig. 2a).

As in our previous results 10,11 , set size affected asymptotic discriminability $(\lambda)$, but not processing speed $(\beta$ or $\delta$ ), at each eccentricity. As set size increased from 1 to 8 , asymptotic discriminability decreased by $0.26 d^{\prime}$ units $(0.15-0.37$ for individual observers) from 1.62 to 1.36 at $4^{\circ}$, and by $0.28 d^{\prime}$ units (0.18-0.59 for individual observers) from 1.93 to 1.65 at $9^{\circ}$. Set size did not affect processing speed, indicating that all items are processed in parallel. The reduction in discriminability with set size is consistent with the fact that distracters engender lateral masking between items, as well as with several 'confusability' accounts of visual search 10,11 . The signal-to-noise ratio decreases with number of distracters, leading to an increased likelihood of a decision error.

The new results concern eccentricity. Although stimulus contrast was kept constant across eccentricities to ensure comparable overall discriminability, asymptotic accuracy was slightly higher at $9^{\circ}$ than $4^{\circ}$. Notably, processing speed was much faster at $9^{\circ}$ than $4^{\circ}$ for every observer, whether the target was presented alone or with distracters. The speed differences were best expressed as an $87 \mathrm{~ms}$ (113, 67 and $87 \mathrm{~ms}$ for individual observers) advantage in intercept for $9^{\circ}(310 \mathrm{vs.} 397 \mathrm{~ms})$. Exponential model fits that did not allocate separate intercept parameters to the different eccentricities produced a lower adjusted- $R^{2}$ ( 0.83 vs. 0.95$)$ for the averaged data as well as for each observer ( 0.79 vs. $0.89,0.80$ vs. 0.88 and 0.79 vs. 0.86 ).

More area and neurons are devoted to the central visual field than to peripheral regions, from retinal ganglion cells to visual cortex $1,4,7$. One explanation of the speed advantage for the periphery could be that integration and processing time increase with the size of the cortical area involved, which is larger at parafoveal than peripheral regions. To evaluate this hypothesis, we magnified the stimuli to evoke a cortical representation with the same size, spatial frequency and orientation difference between the target and distracters for both 
eccentricities12 (Supplementary Methods). Although some aspects of temporal processing are scale invariant (e.g., temporal contrast sensitivity is constant across eccentricity with magnified stimuli12), it is unknown whether all aspects of temporal processing scale with magnification.

At the same eccentricity, discriminability was substantially better for the larger targets stimulating a larger cortical area ( $d^{\prime}$ was 2.7 and 2.6 with 0 and 7 distracters, respectively; ranges 2.3-3.3 and 2.4-3.1 for individual observers), but they were processed $40 \mathrm{~ms}$ slower than the smaller stimuli (38-83 ms for individual observers). This is more surprising given that, for a set contrast, response time increases as a monotonic function of spatial frequency 1,8 , and the larger stimuli had a lower retinal spatial frequency (1.1 vs. 2 c.p.d.). Lateral masking or crowding is unlikely to account for the speed differences (Supplementary Methods).

Magnified stimuli diminished, rather than eliminated, the temporal differences between $4^{\circ}$ and $9^{\circ}$ from 87 (397 vs. 310 ) to 47 (397 vs. 350 ) ms, indicating that the size of the stimulated area does not fully account for the temporal differences. Differences in processing speed across conditions are summarized as the time each condition reaches a given proportion of its asymptote, given by the best-fitting exponential. When accuracy was normalized by asymptote (Fig. 2b), processing speed for $9^{\circ}$ magnified stimuli was intermediate: faster than size-matched stimuli at $4^{\circ}$ but slower than unmagnified stimuli at $9^{\circ}$.

These results provide the first behavioral evidence that either increasing eccentricity or stimulating larger cortical regions engenders slower processing. The results are consistent with neurophysiological findings: (i) scaling increases the ratio of P:M cells at a given eccentricity7; the larger ratio seems to slow processing of magnified relative to smaller stimuli at $9^{\circ}$, and it may be partly responsible for offsetting the observed speed differences between size-matched stimuli at $4^{\circ}$ and $9^{\circ}$; and (ii) propagation of intracortical potentials may slow integration and processing time. For instance, visual stimulation outside the classical receptive field (CRF) in V1 induces weak, longer-latency EPSPs, which scale with distance from the CRF, suggesting that integration of visual activation is spread by slow horizontal connections 13. As computations necessary for orientation discrimination can occur in V1, propagation of horizontal connections in this area may be slow enough to account for a longer processing time on the order of tenths of milliseconds14.

The observed speed advantage for more eccentric locations, although diminished with equated cortical size, was larger than expected from neurophysiology $4^{-} 6$. Nevertheless, real stimuli are not magnified to equate cortical area and are likely to engender sizeable time course differences, on the order of $90 \mathrm{~ms}$ even within a relatively small eccentricity range $\left(\right.$ e.g., $\left.4-9^{\circ}\right)$. This difference is substantial on the timescale of visual processing, both neurophysiologically and behaviorally. Indeed, the observed differences are comparable to estimates of the total time of a fast feedforward sweep of activity, from retinal ganglion cells to parietal cortex in non-human primates6. Behaviorally, for example, complex processing of natural scenes is achieved $150 \mathrm{~ms}$ after stimulus onset 15 . The magnitude of the eccentricity effect is likely to have significant perceptual consequences and will need to be addressed by theories of visual processing.

\section{Supplementary Material}

Refer to Web version on PubMed Central for supplementary material. 


\section{Acknowledgments}

We thank J.M. Hupé, M. Landy, P. Lennie, L. Maloney, D. Pelli and R. Shapley for discussions. A National Science Foundation (BCS-9910734/HCP] grant to M.C. and a National Institute of Mental Health (MH57458) grant to B.M. supported this study.

\section{References}

1. DeValois, RL.; DeValois, KK. Spatial Vision. New York: Oxford Univ. Press; 1988.

2. Hartmann E, Lachenmayr B, Brettel H. Vision Res. 1979; 19:1019-1023. [PubMed: 532115]

3. McKee SP, Nakayama K. Vision Res. 1984; 24:25-32. [PubMed: 6695503]

4. Lennie P. Perception. 1998; 27:889-935. [PubMed: 10209632]

5. Schmolesky MT, et al. Neurophysiology. 1998; 79:3272-3278. [PubMed: 9636126]

6. Lamme VA, Roelfsema PR. Trends Neurosci. 2000; 23:571-579. [PubMed: 11074267]

7. Azzopardi P, Jones KE, Cowey A. Vision Res. 1999; 39:2179-2189. [PubMed: 10343800]

8. Breitmeyer, BG. Visual Masking. New York: Oxford Univ. Press; 1984.

9. Reed A. Science. 1973; 181:574-576. [PubMed: 17777808]

10. McElree B, Carrasco M. J. Exp. Psychol. Hum. Percept. Perform. 1999; 25:1517-1539. [PubMed: 10641310]

11. Carrasco M, McElree B. PNAS. 2001; 98:5363-5367. [PubMed: 11309485]

12. Virsu V, Rovamo J, Laurinen P, Nasanen R. Vision Res. 1982; 22:1211-1217. [PubMed: 7147732]

13. Bringuier V, Chavane F, Glaeser L, Fregnac Y. Science. 1999; 283:695-699. [PubMed: 9924031]

14. Girard P, Hupé JM, Bullier J. J. Neurophysiol. 2001; 85:1328-1331. [PubMed: 11248002]

15. Rousselet GA, Fabre-Thorpe M, Thorpe SJ. Nat. Neurosci. 2002; 5:629-630. [PubMed: 12032544] 


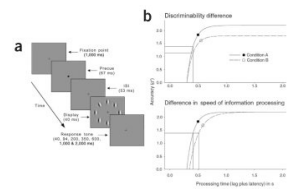

Figure 1.

SAT methodology and hypothetical functions. (a) Sequence of events in a single trial. The stimuli were randomly presented at 8 equidistant locations from a fixation point on an at either 4 or $9^{\circ}$ eccentricity. The interval between the cue onset and the stimulus offset was brief enough to prevent goal or target directed eye movements 1,8 . Observers were required to respond within $300 \mathrm{~ms}$ of the tone whether the target was tilted to the right or the left. Feedback was provided after each trial and block. (b) Hypothetical SAT functions plotted in $d^{\prime}$ units $(\sqrt{2}$ of the standard normal deviate of the probability of correctly judging the target's orientation) versus processing time (time of the response cue plus observer's average latency to respond). Top, expected pattern if the functions differ in asymptotic accuracy, but are associated with the same intercept and proportional rate of information accrual. Bottom, one expected pattern if the functions differ in speed of information accrual only. The intercept $(d$ ' $=0$ ) measures the minimal time needed for above-chance discrimination. The rate of rise of the function indexes the rate of information accrual directly9'10. A difference in either rate or intercept will result in disproportional SAT dynamics, so that the functions will reach a given proportion of their respective asymptotes at different times. The lines that intersect the ordinate and abscissa show the time when the functions reach the $1-1 / e(63 \%)$ point. The circles show the corresponding reaction time (RT) points in SAT coordinates, illustrating that RT differences can arise from differences in discriminability (top) or dynamics (bottom). The position of the RT points on the corresponding SAT functions are determined by the decision criteria that an observer uses to balance speed and accuracy. 


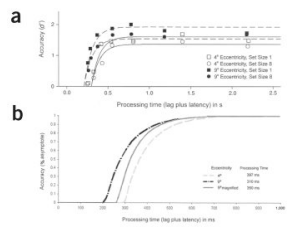

Figure 2.

SAT results and speed of visual processing. (a) Average discrimination accuracy (in $d^{\prime}$ ) as a function of processing time. Smooth functions show the best-fitting exponential model (equation 1 ) for $4^{\circ}$ (solid lines) and $9^{\circ}$ (dashed lines) conditions. The best-fitting model allocated a separate asymptotic parameter $(\lambda)$ to each of the 4 conditions, a common rate $(\beta)$ parameter, and one intercept $(\delta)$ parameter to $4^{\circ}$ and another to $9^{\circ}$ eccentricity. Adjusted- $R^{2}$ was 0.95 . (b) Temporal dynamics of feature searches. Accuracy normalized by asymptote $(\lambda)$ isolates the differences in processing speed for feature searches at $4^{\circ}, 9^{\circ}$ and $9^{\circ}$ magnified (average over set sizes 1 and 8). Differences in processing speed are illustrated by plotting the time at which each condition reaches a given proportion of its asymptote, using the best fitting exponential parameters (Supplementary Methods). 